\title{
On the Cauchy Problem for Von Neumann-Landau Wave Equation
}

\author{
Chuangye Liu', Minmin Liu² \\ ${ }^{1}$ Laboratory of Nonlinear Analysis, Department of Mathematics, Central China Normal University, Wuhan, \\ China \\ ${ }^{2}$ School of Science, Wuhan Institute of Technology, Wuhan, China \\ Email: chuangyeliu1130@126.com, ocbmml@126.com
}

Received 21 October 2014; revised 16 November 2014; accepted 11 December 2014

Copyright (C) 2014 by authors and Scientific Research Publishing Inc.

This work is licensed under the Creative Commons Attribution International License (CC BY).

http://creativecommons.org/licenses/by/4.0/

(c) () Open Access

\section{Abstract \\ In present paper we prove the local well-posedness for Von Neumann-Landau wave equation by the T. Kato's method.}

\section{Keywords}

Von Neumann-Landau Wave Equation, Strichartz Estimate, Cauchy Problem

\section{Introduction}

For the stationary Von Neumann-Landau wave equation, Chen investigated the Dirichlet problems [1], where the generalized solution is studied by Function-analytic method. The present paper is related to the Cauchy problem: the Von Neumann-Landau wave equation

$$
\left\{\begin{array}{l}
i \partial_{t} u=\left(-\Delta_{x}+\Delta_{y}\right) u+f(u) \\
u(0, x, y)=u_{0}(x, y)
\end{array},\right.
$$

where $\Delta_{x}=\sum_{i=1}^{n} \frac{\partial^{2}}{\partial x_{i}^{2}}$ for $x=\left(x_{1}, \cdots, x_{n}\right) \in \mathbb{R}^{n}, u(t, x, y)$ is an unknown complex valued function on $\mathbb{R}^{1+2 n}$ and $f$ is a nonlinear complex valued function.

If the plus "+" is replaced by the minus "-" on right hand in Equation (1), then the resulted equation is the Schrödinger equation. For the Schrödinger equation, the well-posedness problem is investigated for various nonlinear terms $f$. In terms of the nonlinear terms $f$, the problem (1) can be divided into the subcritical case and the critical case for $H^{1}$ solutions. We are concerned with the subcritical case and obtain a local well- 
posedness result by the T. Kato's method.

The paper is organized as follows. Section 2 contains the list of assumptions on the interaction term $f$ and the main result is presented. Section 3 is concerned with the Strichartz estimates. Finally, in Section 4, the main result is proved.

\section{Statement of the Main Result}

In this section we list the assumptions on the interaction term $f$ and state the main result. Firstly, we recall that the definition of admissible pair [2].

Definition 2.1. Fix $d=2 n, n \geq 1$. We say that a pair $(q, r)$ of exponents is admissible if

$$
\frac{2}{q}=d\left(\frac{1}{2}-\frac{1}{r}\right)
$$

and

$$
2 \leq r \leq \frac{2 d}{d-2}(2 \leq r<\infty, \text { if } d=2) .
$$

Remark 2.1. The pairs $(\infty, 2)$ is always admissible, so is the $\left(2, \frac{2 d}{d-2}\right)$ if $d>2$. The two pairs are called the endpoint cases.

Secondly, let $f \in C(\mathbb{C}, \mathbb{C})$ satisfy

$$
f(0)=0 \text {, }
$$

and

$$
|f(u)-f(v)| \leq K(M)|u-v|,
$$

for all $u, v \in \mathbb{C}$ such that $|u|,|v| \leq M$, with

$$
K(t) \leq C_{1}\left(1+t^{\alpha}\right), 0<\alpha<\frac{4}{d-2},
$$

where $C_{1}$ is a constant independent of $t$. Set

$$
f(u)(x)=f(u(x)),
$$

for all measurable function $u$ and a.e. $x \in \mathbb{R}^{1+2 n}$.

Finally, let us make the notion of solution more precise.

Definition 2.2. Let $I$ be an interval such that $0 \in I$. We say that $u$ is a strong $H^{1}$-solution of (1) on $I$ if $u \in C\left(I, H^{1}\left(\mathbb{R}^{d}\right)\right)$ satisfies the integral equation

$$
u(t)=\mathrm{e}^{-i t L} u_{0}-i \int_{0}^{t} \mathrm{e}^{-i(t-s) L} f(u(s)) \mathrm{d} s,
$$

for all $t \in I$, where $L=:-\Delta_{x}+\Delta_{y}$.

The main result is the following theorem:

Theorem 1. Suppose $n \geq 1$. Let $f \in C(\mathbb{C}, \mathbb{C})$ satisfy (4)-(6). If $f$ (considered as a function $\mathbb{R}^{2} \rightarrow \mathbb{R}^{2}$ ) is of class $C^{1}$, then the Cauchy problem (1) is locally well posed in $H^{1}\left(\mathbb{R}^{d}\right)$. More specially, the following properties hold:

(i) For any $R>0$ there exists a time $T=T(d, \alpha, R)>0$ and constant $c=c(d, \alpha)$ such that for each $u_{0}$ in the ball $B_{R}:=\left\{\varphi \in H^{1}\left(\mathbb{R}^{d}\right):\|\varphi\|_{H^{1}\left(\mathbb{R}^{d}\right)} \leq R\right\}$ there exists a unique strong $H^{1}$-solution $u$ to the Equation (1) in $C\left([-T, T], H^{1}\left(\mathbb{R}^{d}\right)\right)$ such that 


$$
\|u\|_{L^{\infty}\left((-T, T), H^{1}\left(\mathbb{R}^{d}\right)\right)}+\|u\|_{L^{q}\left((-T, T), W^{1, r}\left(\mathbb{R}^{d}\right)\right)} \leq c\left\|u_{0}\right\|_{H^{1}\left(\mathbb{R}^{d}\right)},
$$

where $r=\alpha+2$, and $(q, r)$ is an admissible pair.

(ii) The map $u_{0} \mapsto u$ is continuous from $B_{R}$ to $C\left([-T, T], H^{1}\left(\mathbb{R}^{d}\right)\right)$;

(iii) For every $u_{0} \in H^{1}\left(\mathbb{R}^{d}\right)$, the unique solution $u$ is defined on a maximal interval $\left(-T_{\min }, T_{\max }\right)$, with $T_{\text {max }}=T_{\max }\left(u_{0}\right) \in(0, \infty]$ and $T_{\text {min }}=T_{\text {min }}\left(u_{0}\right) \in(0, \infty]$;

(iv) There is the blowup alternative: If $T_{\max }<\infty$, then $\|u(t)\|_{H^{1}\left(\mathbb{R}^{d}\right)} \rightarrow+\infty$ as $t / T_{\max }$ (respectively, if $T_{\min }<\infty$, then $\|u(t)\|_{H^{1}\left(\mathbb{R}^{d}\right)} \rightarrow+\infty$ as $\left.t \searrow-T_{\min }\right)$.

Remark 2.2. It follows from Strichartz estimates that

$$
u \in L_{l o c}^{\gamma}\left(\left(-T_{\min }, T_{\max }\right), W^{1, \rho}\left(\mathbb{R}^{d}\right)\right),
$$

for any admissible pair $(\gamma, \rho)$.

Remark 2.3. For the Schrödinger equations, the similar results hold [2]. It implies a fact that the ellipticity of the operator $-\Delta_{x}-\Delta_{y}$ is not the key point in the local well-posedness problem.

\section{Strichartz Estimates}

In this subsection, we recall that the Strichartz estimates. Let $(\xi, \eta)$ denote a general Fourier variable in $\mathbb{R}^{2 n}$, $\xi=\left(\xi_{1}, \cdots, \xi_{n}\right), \quad \eta=\left(\eta_{1}, \cdots, \eta_{n}\right)$. Let $L=:-\Delta_{x}+\Delta_{y}$, then by Fourier transform(denoting by $\mathcal{F}$ or $\wedge$ ) we have

$$
L u=\mathcal{F}^{-1}\left(\left(|\xi|^{2}-|\eta|^{2}\right) \hat{u}\right),
$$

for any $u \in H^{2}\left(\mathbb{R}^{2 n}\right)$. It is easy to verify that the $L$ is a self-adjoint unbounded operator on $L^{2}\left(\mathbb{R}^{2 n}\right)$ with the domain $H^{2}\left(\mathbb{R}^{2 n}\right)$. Then, by Stone theorem we see that $e^{i t L}$ is an unitary group on $L^{2}\left(\mathbb{R}^{2 n}\right)$. Moreover, $\mathrm{e}^{\mathrm{i} t L}$ can be expressed explicitly by Fourier transform.

$$
\mathrm{e}^{i t L} \varphi=\mathcal{F}^{-1}\left(\mathrm{e}^{i t\left(|\xi|^{2}-|\eta|^{2}\right)} \hat{\varphi}\right)
$$

for any $\varphi \in L^{2}\left(\mathbb{R}^{2 n}\right)$. By the direct compute, we conclude

$$
\left(\mathrm{e}^{i t L} \varphi\right)(x, y)=\frac{1}{(4 \pi i|t|)^{n}} \int_{\mathbb{R}^{2 n}} \mathrm{e}^{\frac{-i\left|x-x^{\prime}\right|^{2}}{4 t}} \mathrm{e}^{\frac{i\left|y-y^{\prime}\right|^{2}}{4 t}} \varphi\left(x^{\prime}, y^{\prime}\right) \mathrm{d} x^{\prime} \mathrm{d} y^{\prime} .
$$

The following result is the fundamental estimate for $\mathrm{e}^{i t L}$.

Lemma 1. If $p \in[1,2]$ and $t \neq 0$, then $\mathrm{e}^{i t L}$ maps $L^{p}\left(\mathbb{R}^{d}\right)$ continuously to $L^{p^{\prime}}\left(\mathbb{R}^{d}\right)$ and

$$
\left\|\mathrm{e}^{i t L} \varphi\right\|_{L^{p^{\prime}}\left(\mathbb{R}^{d}\right)} \leq(4 \pi|t|)^{-d\left(\frac{1}{p}-\frac{1}{2}\right)}\|\varphi\|_{L^{p}\left(\mathbb{R}^{d}\right)}
$$

where $p^{\prime}$ is the dual exponent of $p$, defined by the formula $\frac{1}{p}+\frac{1}{p^{\prime}}=1$.

Proof. For the proof please see [3] or [4]. $\square$ [5].

The following estimates, known as Strichartz estimates, are key points in the method introduced by T. Kato

Lemma 2. Let $(q, r)$ and $(\tilde{q}, \tilde{r})$ be any admissible exponents. Then, we have the homogeneous Strichartz 
estimate

$$
\left\|\mathrm{e}^{i t L} \varphi\right\|_{L^{q}\left(\mathbb{R}, L^{r}\left(\mathbb{R}^{d}\right)\right)} \lesssim_{d, q, r}\|\varphi\|_{L^{2}\left(\mathbb{R}^{d}\right)},
$$

the dual homogeneous Strichartz estimate

$$
\left\|\int_{\mathbb{R}} \mathrm{e}^{-i t L} \phi(t) \mathrm{d} t\right\|_{L^{2}\left(\mathbb{R}^{d}\right)} \lesssim_{d, q, r}\|\phi\|_{L^{q^{\prime}}\left(\mathbb{R}, L^{\prime}\left(\mathbb{R}^{d}\right)\right)},
$$

and the inhomogeneous Strichartz estimate

$$
\left\|\int_{t_{0}}^{t} \mathrm{e}^{i(t-s) L} \phi(s) \mathrm{ds}\right\|_{L^{q}\left(J, L^{r}\left(\mathbb{R}^{d}\right)\right)} \lesssim_{d, q, r, \tilde{q}, \tilde{r}}\|\phi\|_{L^{\tilde{q}^{\prime}}\left(J, L^{\tilde{r}^{\prime}}\left(\mathbb{R}^{d}\right)\right)},
$$

for any interval $J$ and real number $t_{0}$.

Proof. For the proof please see [3] or [4] in the non-endpoint case. On the other hand, the proof in the endpoint case follows from the theorem 1.2 in [6] and the lemma 1 in the present paper. $\square$

\section{The Proof of Theorem}

Proof. Let $\chi \in C_{0}^{\infty}\left(\mathbb{R}^{2}\right)$ be such that $\chi(z)=1$ for $|z| \leq 1$ and $\chi(z)=0$ for $|z| \geq 2$. Setting

$$
\begin{aligned}
& f_{1}(z)=\chi(z) f(z), \\
& f_{2}(z)=(1-\chi(z)) f(z),
\end{aligned}
$$

one easily verifies that for any $z, w \in \mathbb{C}$

$$
\begin{aligned}
& \left|f_{1}(z)-f_{1}(w)\right| \lesssim_{\alpha}|z-w|, \\
& \left|f_{2}(z)-f_{2}(w)\right| \lesssim_{\alpha}\left(|z|^{\alpha}+|w|^{\alpha}\right)|z-w| .
\end{aligned}
$$

Set $f_{l}(u)(x)=f_{l}(u(x))$ for $l=1,2$. Using (17), we deduce from Hölder's inequality that

$$
\begin{aligned}
& \left\|f_{1}(u)-f_{1}(v)\right\|_{L^{2}\left(\mathbb{R}^{d}\right)} \lesssim_{\alpha}\|u-v\|_{L^{2}\left(\mathbb{R}^{d}\right)}, \\
& \left\|f_{2}(u)-f_{2}(v)\right\|_{L^{\prime}\left(\mathbb{R}^{d}\right)} \lesssim_{\alpha}\left(\|u\|_{L^{r}\left(\mathbb{R}^{d}\right)}^{\alpha}+\|v\|_{L^{r}\left(\mathbb{R}^{d}\right)}^{\alpha}\right)\|u-v\|_{L^{r}\left(\mathbb{R}^{d}\right)} .
\end{aligned}
$$

And it follows from Remark 1.3.1 (vii) in [2] that

$$
\begin{aligned}
& \left\|\nabla f_{1}(u)\right\|_{L^{2}\left(\mathbb{R}^{d}\right)} \lesssim_{\alpha}\|\nabla u\|_{L^{2}\left(\mathbb{R}^{d}\right)}, \\
& \left\|\nabla f_{2}(u)\right\|_{L^{r}\left(\mathbb{R}^{d}\right)} \lesssim_{\alpha}\|u\|_{L^{r}\left(\mathbb{R}^{d}\right)}^{\alpha}\|\nabla u\|_{L^{r}\left(\mathbb{R}^{d}\right)} .
\end{aligned}
$$

We now proceed in four steps.

Step 1. Proof of (i). Fix $A, T>0$, to be chosen later, and let $r=\alpha+2, q$ be such that $(q, r)$ is an admissible pair, and set $I=(-T, T)$. Consider the set

$$
E=\left\{u \in L^{\infty}\left(I, H^{1}\left(\mathbb{R}^{d}\right)\right) \cap L^{q}\left(I, W^{1, r}\left(\mathbb{R}^{d}\right)\right):\|u\|_{L^{\infty}\left(I, H^{1}\left(\mathbb{R}^{d}\right)\right)} \leq A,\|u\|_{L^{q}\left(I, W^{1, r}\left(\mathbb{R}^{d}\right)\right)} \leq A\right\},
$$

equipped with the distance

$$
d(u, v)=\|u-v\|_{L^{\infty}\left(I, L^{2}\left(\mathbb{R}^{d}\right)\right)}+\|u-v\|_{L^{q}\left(I, L^{r}\left(\mathbb{R}^{d}\right)\right)} .
$$

We claim that $(E, d)$ is a complete metric space. Indeed, let $\left\{u_{k}\right\}_{k \geq 1} \subset E$ be a Cauchy sequence. Clearly, $\left\{u_{k}\right\}_{k \geq 1}$ is also a Cauchy sequence in $L^{\infty}\left(I, L^{2}\left(\mathbb{R}^{d}\right)\right)$ and $L^{q}\left(I, L^{r}\left(\mathbb{R}^{d}\right)\right)$. In particular, there exists a 
function $u \in L^{\infty}\left(I, L^{2}\left(\mathbb{R}^{d}\right)\right) \cap L^{q}\left(I, L^{r}\left(\mathbb{R}^{d}\right)\right)$ such that $u_{k} \rightarrow u$ in $L^{\infty}\left(I, L^{2}\left(\mathbb{R}^{d}\right)\right)$ and $L^{q}\left(I, L^{r}\left(\mathbb{R}^{d}\right)\right)$ as $k \rightarrow \infty$. Applying theorem 1.2.5 in [2] twice, we conclude that

$$
u \in L^{\infty}\left(I, H^{1}\left(\mathbb{R}^{d}\right)\right) \cap L^{q}\left(I, W^{1, r}\left(\mathbb{R}^{d}\right)\right),
$$

and that

$$
\begin{aligned}
& \|u\|_{L^{\infty}\left(I, H^{1}\left(\mathbb{R}^{d}\right)\right)} \leq \liminf _{k \rightarrow \infty}\left\|u_{k}\right\|_{L^{\infty}\left(I, H^{1}\left(\mathbb{R}^{d}\right)\right)} \leq A, \\
& \|u\|_{L^{q}\left(I, W^{1, r}\left(\mathbb{R}^{d}\right)\right)} \leq \liminf _{k \rightarrow \infty}\left\|u_{k}\right\|_{L^{q}\left(I, W^{1, r}\left(\mathbb{R}^{d}\right)\right)} \leq A ;
\end{aligned}
$$

thus, $u_{k} \rightarrow u$ in $E$ as $k \rightarrow \infty$.

Taking up any $u, v \in E$. Since $f_{1}$ is continuous $L^{2}\left(\mathbb{R}^{d}\right) \rightarrow L^{2}\left(\mathbb{R}^{d}\right)$, it follows that $f_{1}(u): I \rightarrow L^{2}\left(\mathbb{R}^{d}\right)$ is measurable, and we deduce easily that $f_{1}(u) \in L^{\infty}\left(I, L^{2}\left(\mathbb{R}^{d}\right)\right)$. Similarly, since $f_{2}$ is continuous $L^{r}\left(\mathbb{R}^{d}\right) \rightarrow L^{r^{\prime}}\left(\mathbb{R}^{d}\right)$, we see that $f_{2}(u) \in L^{q}\left(I, L^{r^{\prime}}\left(\mathbb{R}^{d}\right)\right)$. Using inequalities (18) and (19) and Remark 1.2.2 (iii) in [2], We deduce the following:

$$
\begin{aligned}
& f_{1}(u) \in L^{\infty}\left(I, H^{1}\left(\mathbb{R}^{d}\right)\right), f_{2}(u) \in L^{q}\left(I, w^{1, r^{\prime}}\left(\mathbb{R}^{d}\right)\right), \\
& \left\|f_{1}(u)\right\|_{L^{\infty}\left(I, H^{1}\left(\mathbb{R}^{d}\right)\right)} \lesssim_{\alpha}\|u\|_{\left.L^{\infty}\left(I, H^{1}\left(\mathbb{R}^{d}\right)\right)\right)} \\
& \left\|f_{2}(u)\right\|_{L^{q}\left(I, w^{1, r^{\prime}}\left(\mathbb{R}^{d}\right)\right)} \lesssim_{\alpha}\|u\|_{\left.L^{\infty}\left(I, L^{r}\left(\mathbb{R}^{d}\right)\right)\right)}\|u\|_{L^{q}\left(I, W^{1, r}\left(\mathbb{R}^{d}\right)\right)},
\end{aligned}
$$

and

$$
\begin{aligned}
& \left\|f_{1}(u)-f_{1}(v)\right\|_{L^{\infty}\left(I, L^{2}\left(\mathbb{R}^{d}\right)\right)} \lesssim_{\alpha}\|u-v\|_{L^{\infty}\left(I, L^{2}\left(\mathbb{R}^{d}\right)\right)}, \\
& \left\|f_{2}(u)-f_{2}(v)\right\|_{L^{q}\left(I, I^{\prime}\left(\mathbb{R}^{d}\right)\right)} \lesssim_{\alpha}\left(\|u\|_{\left.L^{\infty}\left(I, L^{r}\left(\mathbb{R}^{d}\right)\right)\right)}^{\alpha}+\|v\|_{L^{\infty}\left(I, L^{r}\left(\mathbb{R}^{d}\right)\right)}^{\alpha}\right)\|u-v\|_{L^{q}\left(I, L^{r}\left(\mathbb{R}^{d}\right)\right)} \cdot
\end{aligned}
$$

Using the embedding $H^{1}\left(\mathbb{R}^{d}\right) \hookrightarrow L^{r}\left(\mathbb{R}^{d}\right)$ and Hölder's inequality in time, we deduce from the above estimates that

$$
\left\|f_{1}(u)\right\|_{L^{1}\left(I, H^{1}\left(\mathbb{R}^{d}\right)\right)}+\left\|f_{2}(u)\right\|_{L^{q^{\prime}}\left(I, W^{1, r^{\prime}}\left(\mathbb{R}^{d}\right)\right)} \lesssim_{d, \alpha}\left(T+T^{\frac{q-q^{\prime}}{q q^{\prime}}}\right)\left(1+A^{\alpha}\right) A
$$

and

$$
\left\|f_{1}(u)-f_{1}(v)\right\|_{L^{1}\left(I, L^{2}\left(\mathbb{R}^{d}\right)\right)}+\left\|f_{2}(u)-f_{2}(v)\right\|_{L^{q^{\prime}}\left(I, L^{\prime}\left(\mathbb{R}^{d}\right)\right)} \lesssim_{d, \alpha}\left(T+T^{\frac{q-q^{\prime}}{q q^{\prime}}}\right)\left(1+A^{\alpha}\right) d(u, v) .
$$

Given $u_{0} \in H^{1}\left(\mathbb{R}^{d}\right)$. For any $u \in E$, let $\mathcal{H}(u)$ be defined by

$$
\mathcal{H}(u)(t)=\mathrm{e}^{-i t L} u_{0}-i \int_{0}^{t} \mathrm{e}^{-i(t-s) L} f(u(s)) \mathrm{d} s .
$$

It follows from (22) and Strichartz estimates (lemma 2) that

$$
\mathcal{H}(u) \in C\left([-T, T], H^{1}\left(\mathbb{R}^{d}\right)\right) \cap L^{q}\left((-T, T), W^{1, r}\left(\mathbb{R}^{d}\right)\right),
$$

and

$$
\|\mathcal{H}(u)\|_{L^{\infty}\left(I, H^{1}\left(\mathbb{R}^{d}\right)\right)}+\|\mathcal{H}(u)\|_{L^{q}\left(I, W^{1, r}\left(\mathbb{R}^{d}\right)\right)} \leq C_{1}(d, \alpha)\left[\left\|u_{0}\right\|_{H^{1}\left(\mathbb{R}^{d}\right)}+\left(T+T^{\frac{q-q^{\prime}}{q q^{\prime}}}\right)\left(1+A^{\alpha}\right) A\right] .
$$


Also, we deduce from (23) that

$$
\|\mathcal{H}(u)-\mathcal{H}(v)\|_{L^{\infty}\left(I, L^{2}\left(\mathbb{R}^{d}\right)\right)}+\|\mathcal{H}(u)-\mathcal{H}(v)\|_{L^{q}\left(I, L^{r}\left(\mathbb{R}^{d}\right)\right)} \leq C_{1}(d, \alpha)\left(T+T^{\frac{q-q^{\prime}}{q q^{\prime}}}\right)\left(1+A^{\alpha}\right) d(u, v) .
$$

Finally, note that $q>q^{\prime}$. We now proceed as follows. For any $R \geq\left\|u_{0}\right\|_{H^{1}\left(\mathbb{R}^{d}\right)}$, we set $A=2 C_{1}(d, \alpha) R$, and we let $T=T(d, \alpha, R)$ be the unique positive number so that

$$
C_{1}(d, \alpha)\left(T+T^{\frac{q-q^{\prime}}{q q^{\prime}}}\right)\left(1+A^{\alpha}\right)=\frac{1}{2} .
$$

It then follows from (26) and (28) that for any $u_{0} \in B_{R}$

$$
\|\mathcal{H}(u)\|_{L^{\infty}\left(I, H^{1}\left(\mathbb{R}^{d}\right)\right)}+\|\mathcal{H}(u)\|_{L^{q}\left(I, W^{1, r}\left(\mathbb{R}^{d}\right)\right)} \leq C_{1}(d, \alpha)\left\|u_{0}\right\|_{H^{1}\left(\mathbb{R}^{d}\right)}+\frac{1}{2} A \leq C_{1}(d, \alpha) R+\frac{1}{2} A=A .
$$

Thus, $\mathcal{H}(u) \in E$ and by (27) we obtain

$$
d(\mathcal{H}(u), \mathcal{H}(v)) \leq \frac{1}{2} d(u, v)
$$

In particular, $\mathcal{H}$ is a strict contraction on $E$. By Banach's fixed-point theorem, $\mathcal{H}$ has a unique fixed point $u \in E$; that is $u$ satisfies (8). By (25), $u=\mathcal{H}(u) \in C\left([-T, T], H^{1}\left(\mathbb{R}^{d}\right)\right)$. By the definition 2.2, we con- clude that $u$ is a strong $H^{1}$-solution of (1) on $[-T, T]$. Note that $T(d, \alpha, R)$ is decreasing on $R$, then the estimate (9) holds for $c=2 C_{1}(d, \alpha)$ by letting $R=\left\|u_{0}\right\|_{H^{1}\left(\mathbb{R}^{d}\right)}$ in (29).

For uniqueness, assume that $u, v$ are two strong $H^{1}$-solution of (1) on $[-T, T]$ with the same initial value $u_{0}$. Then, we have

$$
u(t)-v(t)=-i \int_{0}^{t} \mathrm{e}^{-i(t-s) L}[f(u(s))-f(v(s))] \mathrm{d} s .
$$

For simplicity, we set

$$
w_{l}(t)=-i \int_{0}^{t} \mathrm{e}^{-i(t-s) L}\left[f_{l}(u(s))-f_{l}(v(s))\right] \mathrm{d} s,
$$

for $l=1,2$, and $w=u-v$. For any interval $J \subset(-T, T)$, by (18) and Strichartz estimates (16), then we obtain

$$
\left\|w_{1}\right\|_{L^{\infty}\left(J, L^{2}\left(\mathbb{R}^{d}\right)\right)}+\left\|w_{1}\right\|_{L^{q}\left(J, L^{r}\left(\mathbb{R}^{d}\right)\right)} \lesssim_{d, \alpha}\left\|f_{1}(u)-f_{1}(v)\right\|_{L^{1}\left(J, L^{2}\left(\mathbb{R}^{d}\right)\right)} \lesssim_{d, \alpha}\|w\|_{L^{1}\left(J, L^{2}\left(\mathbb{R}^{d}\right)\right)}
$$

Similarly, for $w_{2}$ we have

$$
\begin{aligned}
& \left\|w_{2}\right\|_{L^{\infty}\left(J, L^{2}\left(\mathbb{R}^{d}\right)\right)}+\left\|w_{2}\right\|_{L^{q}\left(J, L^{r}\left(\mathbb{R}^{d}\right)\right)} \lesssim_{d, \alpha}\left\|f_{2}(u)-f_{2}(v)\right\|_{L^{q^{\prime}}\left(J, L^{\prime}\left(\mathbb{R}^{d}\right)\right)} \\
& \lesssim_{d, \alpha}\left(\|u\|_{L^{\infty}\left(I, H^{1}\left(\mathbb{R}^{d}\right)\right)}^{\alpha}+\|v\|_{L^{\infty}\left(I, H^{1}\left(\mathbb{R}^{d}\right)\right)}^{\alpha}\right)\|w\|_{L^{\prime}\left(J, L^{r}\left(\mathbb{R}^{d}\right)\right)} \cdot
\end{aligned}
$$

Note that $w=w_{1}+w_{2}$. Then, it follows from that

$$
\|w\|_{L^{\infty}\left(J, L^{2}\left(\mathbb{R}^{d}\right)\right)}+\|w\|_{L^{q}\left(J, L^{r}\left(\mathbb{R}^{d}\right)\right)} \leq C_{2}(1+B)\left(\|w\|_{L^{1}\left(J, L^{2}\left(\mathbb{R}^{d}\right)\right)}+\|w\|_{L^{q}\left(J, L^{r}\left(\mathbb{R}^{d}\right)\right)}\right)
$$

where the constant $B=\|u\|_{L^{\infty}\left(I, H^{1}\left(\mathbb{R}^{d}\right)\right)}^{\alpha}+\|v\|_{L^{\infty}\left(I, H^{1}\left(\mathbb{R}^{d}\right)\right)}^{\alpha}$ and the constant $C_{2}$ is independent of $J$ by above inequalities. Note that $q^{\prime}<q$, we conclude that $w=0$ by the lemma 4.2.2 in [2]. So $u=v$.

Step 2. Proof of (ii). Suppose that $u_{0}^{(k)} \rightarrow u_{0}$ in $B_{R}$ as $k \rightarrow \infty$. By the part (i), we denote $u_{k}$ and $u$ by 
the unique solution of (1) corresponding to the initial value $u_{0}^{(k)}$ and $u$, respectively. We will show that $u_{k} \rightarrow u$ in $C\left([-T, T], H^{1}\left(\mathbb{R}^{d}\right)\right)$ as $k \rightarrow \infty$. Note that

$$
u_{k}(t)-u(t)=\mathrm{e}^{-i t L}\left(u_{0}^{(k)}-u_{0}\right)+\mathcal{H}\left(u_{k}\right)-\mathcal{H}(u)
$$

and the estimate (29) which implies that (27) holds for $v=u_{k}$. Note that the choosing of the time $T$ in (28), it follows from (27) with (30) that

$$
d\left(u_{k}, u\right) \lesssim_{d, \alpha}\left\|u_{0}^{(k)}-u_{0}\right\|_{L^{2}\left(\mathbb{R}^{d}\right)}+\frac{1}{2} d\left(u_{k}, u\right) .
$$

Hence, we have

$$
\left\|u_{k}-u\right\|_{L^{\infty}\left((-T, T), L^{2}\left(\mathbb{R}^{d}\right)\right)}+\left\|u_{k}-u\right\|_{L^{q}\left((-T, T), L^{r}\left(\mathbb{R}^{d}\right)\right)} \lesssim_{d, \alpha}\left\|u_{0}^{(k)}-u_{0}\right\|_{L^{2}\left(\mathbb{R}^{d}\right)} .
$$

Next, we need to estimate $\left\|\nabla\left(u_{k}-u\right)\right\|_{L^{\infty}\left((-T, T), L^{2}\left(\mathbb{R}^{d}\right)\right)}$. Note that $\nabla$ commutes with $\mathrm{e}^{-i t L}$, and so

$$
\nabla u(t)=\mathrm{e}^{-i t L} \nabla u_{0}-i \int_{0}^{t} \mathrm{e}^{-i(t-s) L} \nabla f(u(s)) \mathrm{d} s .
$$

A similar identity holds for $u_{k}$. We use the assumption $f \in C^{1}\left(\mathbb{R}^{2}, \mathbb{R}^{2}\right)$, which implies that $\nabla f(u)=f^{\prime}(u) \nabla u$, where $f^{\prime}(u)$ is a $2 \times 2$ real matrix. Therefore, we may write

$$
\begin{aligned}
\nabla\left(u_{k}-u\right)(t)= & \mathrm{e}^{-i t L} \nabla\left(u_{0}^{(k)}-u_{0}\right)-i \int_{0}^{t} \mathrm{e}^{-i(t-s) L} f^{\prime}\left(u_{k}\right) \nabla\left(u_{k}-u\right) \mathrm{d} s \\
& -i \int_{0}^{t} \mathrm{e}^{-i(t-s) L}\left(f^{\prime}\left(u_{k}\right)-f^{\prime}(u)\right) \nabla u \mathrm{~d} s .
\end{aligned}
$$

Note that $f_{1}$ and $f_{2}$ are also $C^{1}$, so that $f^{\prime}=f_{1}^{\prime}+f_{2}^{\prime}$, and from (17) we deduce that $\left|f_{1}^{\prime}(z)\right| \leq C_{3}$ and $\left|f_{2}^{\prime}(z)\right| \lesssim_{\alpha}|z|^{\alpha}$ for any $z \in \mathbb{C}$ and some constant $C_{3}$. Therefore, arguing as in Step 1 , we obtain the estimate

$$
\begin{gathered}
\left\|\nabla\left(u_{k}-u\right)\right\|_{L^{\infty}\left((-T, T), L^{2}\left(\mathbb{R}^{d}\right)\right)}+\left\|\nabla\left(u_{k}-u\right)\right\|_{L^{q}\left((-T, T), L^{\prime}\left(\mathbb{R}^{d}\right)\right)} \\
\lesssim_{d, \alpha}\left[\left\|\nabla\left(u_{0}^{(k)}-u_{0}\right)\right\|_{L^{2}\left(\mathbb{R}^{d}\right)}+T\left\|\nabla\left(u_{k}-u\right)\right\|_{L^{\infty}\left((-T, T), L^{2}\left(\mathbb{R}^{d}\right)\right)}\right. \\
+T^{\frac{q-q^{\prime}}{q q^{\prime}}}\left\|u_{k}\right\|_{L^{\infty}\left((-T, T), L^{2}\left(\mathbb{R}^{d}\right)\right)}^{\alpha}\left\|\nabla\left(u_{k}-u\right)\right\|_{L^{q}\left((-T, T), L^{r}\left(\mathbb{R}^{d}\right)\right)} \\
+\left\|\left(f_{1}^{\prime}\left(u_{k}\right)-f_{1}^{\prime}(u)\right) \nabla u\right\|_{L^{1}\left((-T, T), L^{2}\left(\mathbb{R}^{d}\right)\right)} \\
+\left\|\left(f_{2}^{\prime}\left(u_{k}\right)-f_{2}^{\prime}(u)\right) \nabla u\right\|_{\left.L^{q^{\prime}}\left((-T, T), L^{\prime}\left(\mathbb{R}^{d}\right)\right)\right] .}
\end{gathered}
$$

By choosing $T=T(d, \alpha, R)$ as (28) and noting that $u_{k} \in B_{R}$, from (40) we obtain that

$$
\begin{aligned}
& \left\|\nabla\left(u_{k}-u\right)\right\|_{L^{\infty}\left((-T, T), L^{2}\left(\mathbb{R}^{d}\right)\right)}+\left\|\nabla\left(u_{k}-u\right)\right\|_{L^{q}\left((-T, T), L^{r}\left(\mathbb{R}^{d}\right)\right)} \\
& \lesssim_{d, \alpha}\left[\left\|\nabla\left(u_{0}^{(k)}-u_{0}\right)\right\|_{L^{2}\left(\mathbb{R}^{d}\right)}+\left\|\left(f_{1}^{\prime}\left(u_{k}\right)-f_{1}^{\prime}(u)\right) \nabla u\right\|_{L^{1}\left((-T, T), L^{2}\left(\mathbb{R}^{d}\right)\right)}\right. \\
& \quad+\left\|\left(f_{2}^{\prime}\left(u_{k}\right)-f_{2}^{\prime}(u)\right) \nabla u\right\|_{\left.L^{q}\left((-T, T), L^{\prime}\left(\mathbb{R}^{d}\right)\right)\right] .}
\end{aligned}
$$

There, if we prove that 


$$
\left\|\left(f_{1}^{\prime}\left(u_{k}\right)-f_{1}^{\prime}(u)\right) \nabla u\right\|_{L^{1}\left((-T, T), L^{2}\left(\mathbb{R}^{d}\right)\right)}+\left\|\left(f_{2}^{\prime}\left(u_{k}\right)-f_{2}^{\prime}(u)\right) \nabla u\right\|_{L^{q^{\prime}}\left((-T, T), L^{r^{\prime}}\left(\mathbb{R}^{d}\right)\right)} \rightarrow 0,
$$

as $k \rightarrow \infty$, then we have

$$
\left\|\nabla\left(u_{k}-u\right)\right\|_{L^{\infty}\left((-T, T), L^{2}\left(\mathbb{R}^{d}\right)\right)}+\left\|\nabla\left(u_{k}-u\right)\right\|_{L^{q}\left((-T, T), L^{r}\left(\mathbb{R}^{d}\right)\right)} \rightarrow 0,
$$

as $k \rightarrow \infty$, which, combined with (37), yields the desired convergence. we prove (42) by contradiction, and we assume that there exists $\varepsilon_{0}>0$, and a subsequence, which we still denote by $\left\{u_{k}\right\}_{k \geq 1}$ such that

$$
\left\|\left(f_{1}^{\prime}\left(u_{k}\right)-f_{1}^{\prime}(u)\right) \nabla u\right\|_{L^{1}\left((-T, T), L^{2}\left(\mathbb{R}^{d}\right)\right)}+\left\|\left(f_{2}^{\prime}\left(u_{k}\right)-f_{2}^{\prime}(u)\right) \nabla u\right\|_{L^{\prime}\left((-T, T), L^{\prime}\left(\mathbb{R}^{d}\right)\right)} \geq \varepsilon_{0} .
$$

By using (37) and possibly extracting a subsequence, we may assume that $u_{k} \rightarrow u$ a.e. on $(-T, T) \times \mathbb{R}^{d}$ and that there exists $v \in L^{q}\left((-T, T), L^{r}\left(\mathbb{R}^{d}\right)\right)$ such that $\left|u_{k}\right| \leq v$ a.e. on $(-T, T) \times \mathbb{R}^{d}$. In particular, both $\left(f_{1}^{\prime}\left(u_{k}\right)-f_{1}^{\prime}(u)\right) \nabla u$ and $\left(f_{2}^{\prime}\left(u_{k}\right)-f_{2}^{\prime}(u)\right) \nabla u$ converge to 0 a.e. on $(-T, T) \times \mathbb{R}^{d}$. Since

$$
\left|\left(f_{1}^{\prime}\left(u_{k}\right)-f_{1}^{\prime}(u)\right) \nabla u\right| \leq 2 C_{3}|\nabla u| \in L^{1}\left((-T, T), L^{2}\left(\mathbb{R}^{d}\right)\right),
$$

and

$$
\left|\left(f_{2}^{\prime}\left(u_{k}\right)-f_{2}^{\prime}(u)\right) \nabla u\right| \lesssim_{\alpha}\left(\left|u_{k}\right|^{\alpha}+|u|^{\alpha}\right)|\nabla u| \lesssim_{\alpha}\left(v^{\alpha}+|u|^{\alpha}\right)|\nabla u| \in L^{q^{\prime}}\left((-T, T), L^{r^{\prime}}\left(\mathbb{R}^{d}\right)\right),
$$

we obtain from the dominated convergence a contradiction with (44).

Step 3. Proof of (iii). Consider $u_{0} \in H^{1}\left(\mathbb{R}^{d}\right)$ and let

$$
\begin{gathered}
T_{\max }\left(u_{0}\right)=\sup \{T>0 \text { : there exists a solution of (1) on }[0, T]\}, \\
T_{\min }\left(u_{0}\right)=\sup \{T>0 \text { : there exists a solution of }(1) \text { on }[-T, 0]\} .
\end{gathered}
$$

It follows from part (i) there exists a solution

$$
u \in C\left(\left(-T_{\min }, T_{\max }\right), H^{1}\left(\mathbb{R}^{d}\right)\right),
$$

of (1).

Step 4. Proof of (iv). Suppose now that $T_{\max }<\infty$, and assume that there exist $M<\infty$ and a sequence $t_{j} \nearrow T_{\max }$ such that $\left\|u\left(t_{j}\right)\right\|_{H^{1}\left(\mathbb{R}^{d}\right)} \leq M$. Let $k$ be such that $t_{k}+T(d, \alpha, M)>T_{\max }\left(u_{0}\right)$. By part (i), from the initial data $u\left(t_{k}\right)$, one can extend $u$ up to $t_{k}+T(d, \alpha, M)$, which contradicts maximality. Therefore,

$$
\|u(t)\|_{H^{1}\left(\mathbb{R}^{d}\right)} \rightarrow \infty \text {, as } t \nearrow T_{\max } \text {. }
$$

One shows by the same argument that if $T_{\min }<\infty$, then

$$
\|u(t)\|_{H^{1}\left(\mathbb{R}^{d}\right)} \rightarrow \infty \text {, as } t \searrow-T_{\min x} .
$$

This completes the proof. $\square$

\section{Acknowledegments}

We are grateful to the anonymous referee for many helpful comments and suggestions, which have been incorporated into this version of the paper. C. Liu was supported in part by the NSFC under Grants No. 11101171, 11071095 and the Fundamental Research Funds for the Central Universities. And M. Liu was 
supported by science research foundation of Wuhan Institute of Technology under grants No. k201422.

\section{References}

[1] Chen, Z. (2009) Dirichlet Problems for Stationary von Neumann-Landau Wave Equations. Acta Mathematica Scientia, 29, 1225-1232. http://dx.doi.org/10.1016/S0252-9602(09)60099-0

[2] Cazenave, T. (2003) Semilinear Schrödinger Equations, Courant Lecture Notes in Mathematics, 10. New York University, Courant Institute of Mathematical Sciences, AMS.

[3] Tao, T. (2006) Nonlinear Dispersive Equations: Local and Global Analysis. CBMS Regional Conference Series in Mathematics, Vol. 108, American Mathematical Society, Providence.

[4] Linares, F. and Ponce, G. (2009) Introduction to Nonlinear Dispersive Equations.

[5] Kato, T. (1987) On nonlinear Schrödinger Equations. Annales de l’I.H.P. Physique Théorique, 46, 113-129.

[6] Keel, M. and Tao, T. (1998) Endpoint Strichartz Estimates. American Journal of Mathematics, 120, 955-980. http://dx.doi.org/10.1353/ajm.1998.0039 
Scientific Research Publishing (SCIRP) is one of the largest Open Access journal publishers. It is currently publishing more than 200 open access, online, peer-reviewed journals covering a wide range of academic disciplines. SCIRP serves the worldwide academic communities and contributes to the progress and application of science with its publication.

Other selected journals from SCIRP are listed as below. Submit your manuscript to us via either submit@scirp.org or Online Submission Portal.
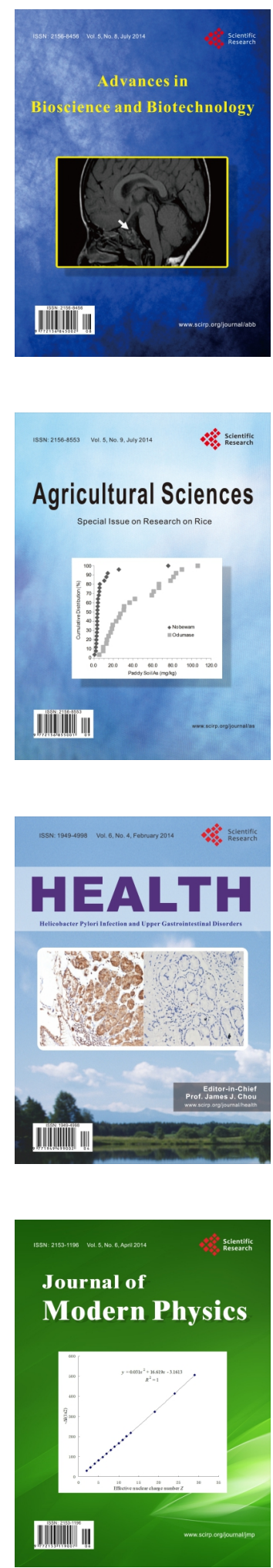
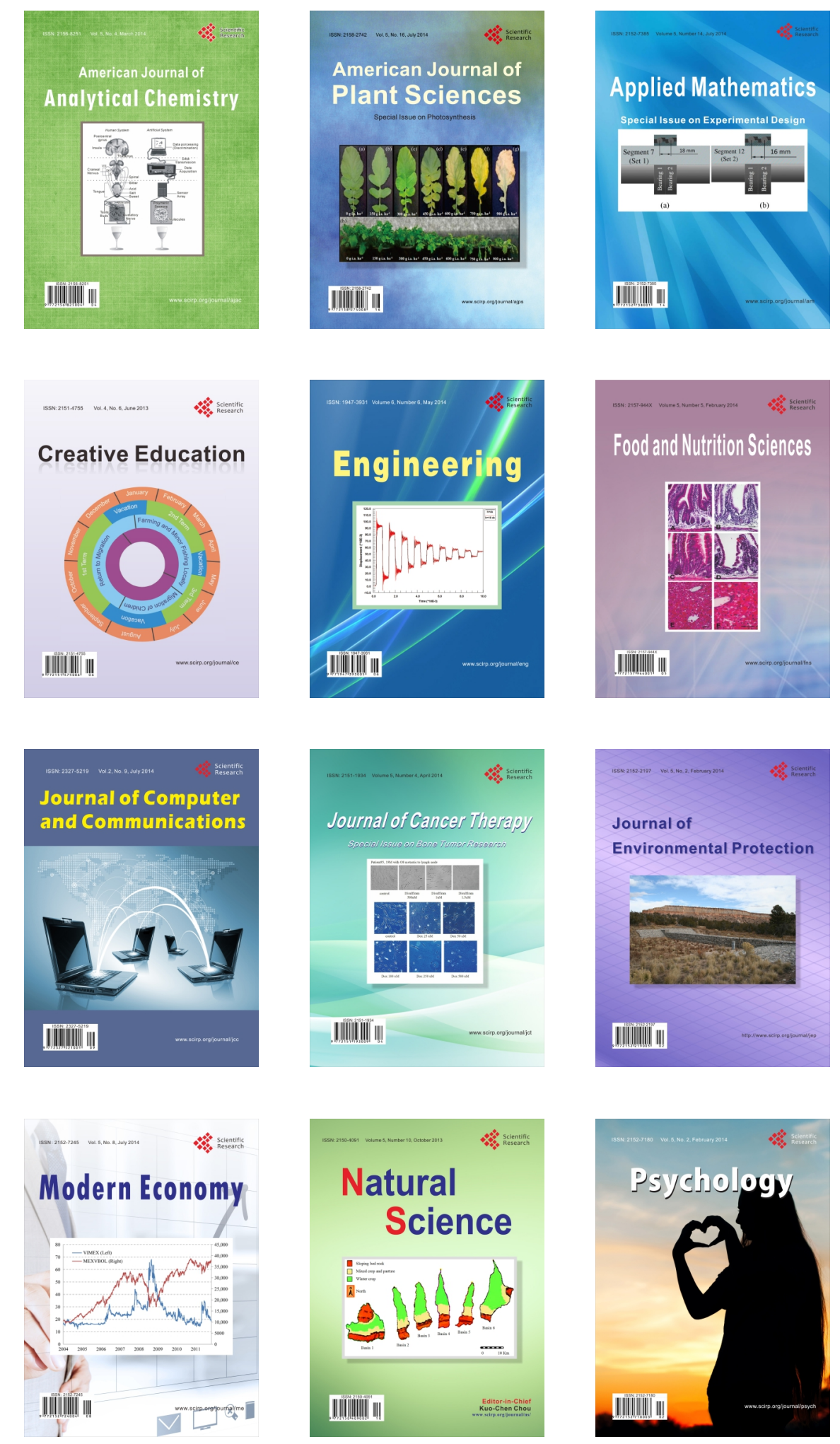